\title{
Validación de la Escala Razones Para Vivir en Consultantes a Salud Mental de la Región Metropolitana de Chile
}

\section{Validation of the Reasons for Living Inventory in Mental Health Patients in the Metropolitan Region of Chile}

\author{
Orietta Echávarri, Susana Morales y Jorge Barros \\ Pontificia Universidad Católica de Chile \\ Iván Armijo, Daniela Larraza, Antonella Longo y Camila Martin \\ Universidad Gabriela Mistral \\ María Paz Maino \\ Pontificia Universidad Católica de Chile \\ Catalina Núñez y Ronit Fischman \\ Instituto Milenio para la Investigación en Depresión y Personalidad
}

\author{
Claudia Moya \\ Marta Szmulewicz \\ Alemka Tomicic
Universidad Diego Portales \\ Universidad San Sebastián Pontificia Universidad Católica de Chile
}

\begin{abstract}
El estudio tuvo como objetivo la validación para Chile de la escala Razones para Vivir (RFL) en una muestra intencionada y consecutiva de 705 consultantes a salud mental en la Región Metropolitana. La RFL evalúa la importancia atribuida a las razones para vivir cuando se piensa en el suicidio. Se analizó la estructura factorial a través de análisis exploratorio y confirmatorio del instrumento original. Se estudió la consistencia interna, la validez de constructo y la validez concurrente y se definieron los parámetros de puntajes. El punto de corte sugerido para Chile es 4,1 , lo que significa que bajo este puntaje hay una mayor probabilidad de riesgo suicida. La sensibilidad es del $62,1 \%$ y la especificidad, $66 \%$, considerado aceptable, dada la alta incidencia de falsos negativos y positivos. El resultado de la evaluación advierte a los tratantes para indagar clínicamente el riesgo suicida detectado en el momento de aplicar el instrumento. Además, para el tratamiento de pacientes con depresión y riesgo suicida, destaca el potencial adaptativo y terapéutico de las razones para mantenerse con vida cuando se está contemplando el suicidio.
\end{abstract}

Palabras clave: suicidio, validación, riesgo, factores de protección

The aim of the study was to validate the Reasons for Living (RFL) Inventory in Chile, using a purposive and consecutive sample of 705 mental health patients in the Metropolitan Region. The RFL scale assesses the importance ascribed to reasons for living when contemplating suicide. The factorial structure of the scale was analyzed through exploratory and confirmatory analysis of the original instrument. Internal consistency, construct validity, and concurrent validity were studied and score parameters were defined. The suggested cut-off point for Chile is 4.1 , which means that lower scores indicate a higher probability of suicide risk. Sensitivity was estimated at $62.1 \%$ and specificity at $66 \%$, which is considered to be acceptable, given the high incidence of false negatives and positives. The

Orietta Echávarri, Susana Morales y María Paz Maino, Departamento de Psiquiatría, Facultad de Medicina, Pontificia Universidad Católica de Chile, e Instituto Milenio para la Investigación en Depresión y Personalidad (MIDAP), Santiago, Chile; Jorge Barros y Marta Szmulewicz, Departamento de Psiquiatría, Facultad de Medicina, Pontificia Universidad Católica de Chile, Santiago, Chile; Iván Armijo, Facultad de Psicología, Universidad Gabriela Mistral, Santiago, Chile; Daniela Larraza, Antonella Longo y Camila Martin, Facultad de Psicología, Universidad Gabriela Mistral, y Centro de Entrenamiento en Psicoterapia y Coaching, Santiago, Chile; Catalina Núñez y Ronit Fischman, MIDAP, Santiago, Chile; Claudia Moya, Facultad de Enfermería, Universidad San Sebastián, y MIDAP, Santiago, Chile; Alemka Tomicic, Facultad de Psicología, Universidad Diego Portales, y MIDAP, Santiago, Chile.

La validación de este instrumento forma parte de un estudio mayor, Proyecto FONDECYT de Iniciación de Investigación $\mathrm{N}^{\circ}$ 11121390, Precisando la evaluación del riesgo suicida: estudio de variables psicosociales asociadas a la conducta suicida en adolescentes y adultos consultantes a salud mental.

Este estudio recibió apoyo del Fondo de Innovación para la Competitividad del Ministerio de Economía, Fomento y Turismo, a través de la Iniciativa Científica Milenio, Proyecto IS130005, y financiamiento del Fondo Nacional de Desarrollo Científico y Tecnológico, Proyecto FONDECYT de Iniciación de Investigación $\mathrm{N}^{\circ} 11121390$. Cuenta con el patrocinio del Departamento de Psiquiatría, Facultad de Medicina, Pontificia Universidad Católica de Chile.

La correspondencia relativa a este artículo debe ser dirigida a Orietta Echávarri, Camino El Alba 12.351, Las Condes, Santiago, Chile. E-mail: oriettaechavarri@gmail.com 
result of the evaluation warns health professionals to clinically investigate the suicide risk detected when administering the instrument. Additionally, results highlight the adaptive and therapeutic potential of the reasons to stay alive for the treatment of depressive patients who are contemplating suicide.

Keywords: suicide, validation, risk, protective factors

En Chile el suicidio se ha constituido en un grave problema de salud pública, reflejado en el aumento de sus tasas (Echávarri, Maino, Fischman, Morales \& Barros, 2015). De acuerdo a la Organización para la Cooperación y el Desarrollo Económico (OCDE), Chile presenta una tasa de 11 por 100.000 habitantes, ocupando el lugar número 21 entre sus países miembros (OECD, 2015). Esto se traduce en que a nivel nacional mueren por suicidio aproximadamente cinco personas al día. Esta cifra es comparable a las muertes por accidentes de tránsito, que llegan en promedio a seis personas al día (OECD, 2015).

De acuerdo a la Organización Mundial de la Salud (OMS, 2012), en el mundo mueren por suicidio cerca de un millón de personas al año, lo que corresponde a una tasa de 16 cada 100.000 habitantes. El suicidio es una de las tres primeras causas de defunción entre las personas de 15 a 44 años y la segunda causa en el grupo de 10 a 24 años. Se estima que del total de suicidios consumados anualmente 250.000 corresponden a menores de 25 años (Organización Panamericana de la Salud [OPS], 2002). Además, se sabe que los intentos de suicidio son hasta 20 veces más frecuentes que los suicidios. Tanto en los suicidios como en los intentos de suicidio su impacto se multiplica más allá de las personas fallecidas, ya que afecta de manera dramática a familiares, amigos y comunidades (World Health Organization [WHO], 2014).

Considerando esta realidad, el Ministerio de Salud de Chile (MINSAL) se ha propuesto, para la década 2011-2020, disminuir en un 15\% la tasa de mortalidad proyectada por suicidio en población de 10 a 19 años. Es decir, la meta de impacto esperada al año 2020 es de 10,2 por 100.000 adolescentes (MINSAL, 2011).

En este contexto, resulta fundamental aportar con estrategias de prevención e intervención que contribuyan a disminuir los suicidios y los intentos de suicidio. Existe un conjunto de información empírica respecto al comportamiento suicida que permite vislumbrar la posibilidad de establecer indicadores para la detección e intervención oportuna por parte de personal capacitado (MINSAL, 2013). En este sentido, se sabe que el $90 \%$ de los suicidios consumados se asocian a cuadros psiquiátricos, como trastornos del ánimo, depresión, ansiedad y abuso de sustancias, entre otros (OMS, 2012; OPS, 2014). Se ha mostrado que el mejor predictor de un suicidio consumado es el intento de suicidio previo (Appleby et al., 1999; Baader-Matthei, Richter \& Mundt, 2004) y que el 80\% de las personas que mueren por suicido da algún tipo de aviso (Bobes García, Giner Ubago \& Saiz Ruiz, 2011). La mayoría de ellas ha visitado a un médico entre tres meses a un año antes de su muerte (Appleby et al., 1999). En consistencia con estos hallazgos, se han desarrollado instrumentos para la evaluación del riesgo suicida enfocados en medir sintomatología y otros factores asociados a este riesgo, como, por ejemplo, síntomas depresivos, desesperanza, impulsividad, ideas suicidas e intentos de suicidio previo (Echávarri, Fischman, Morales, Murillo \& Barros, 2015).

Por otra parte, se ha resaltado la importancia de evaluar factores protectores de manera complementaria a los factores de riesgo. La OPS (2003) señala que cuando hay factores protectores fuertes en una persona con varios factores de riesgo, es posible que no se configuren las condiciones para la ideación o comportamiento suicida. Por otro lado, existe evidencia respecto de que potenciar los factores protectores puede resultar más efectivo que intervenir sobre los factores de riesgo (Borowsky, Resnick, Ireland \& Blum, 1999). Así, ha cobrado relevancia el estudio de los factores disuasivos de la conducta suicida, siendo estos aspectos clave en la pesquisa del riesgo y en la planificación de las intervenciones sociales y terapéuticas (Echávarri, Morales et al., 2015). En este contexto, la escala de Razones para Vivir - Reasons for Living (RFL) — publicada en 1983 por Linehan, Goodstein, Nielsen y Chiles, rescata las razones que evitan que las personas tomen la decisión de quitarse la vida. Indaga sobre creencias, expectativas y capacidades de los sujetos como mediadores en la conducta suicida (Echávarri, Morales et al., 2015; García Valencia et al., 2009). En esta línea, se ha mostrado que un mayor número de razones para vivir se asocia a un bajo riesgo de suicidio y un menor riesgo de reincidencia de intentos de suicidio (Echávarri, Morales et al., 2015; GarcíaValencia et al., 2007).

La escala RFL es un instrumento de auto reporte que ha demostrado ser una contribución significativa de datos empíricos en la toma de decisiones para el tratamiento en adolescentes y adultos con ideación y/o comportamiento suicida (Osman et al., 1999). Dobrov y Thorell (2004) destacan la utilidad de la RFL, tanto en la investigación como en la práctica clínica. A nivel clínico, aporta información útil acerca de los recursos, 
motivos que vinculan a la vida y áreas relevantes a fortalecer en el trabajo psicoterapéutico (Echávarri, Morales et al., 2015; Morales et al., 2015; Taylor, Morales, Zuloaga, Echávarri \& Barros, 2012). A nivel de investigación, diversos estudios muestran propiedades psicométricas sólidas de la escala RFL y su utilidad en la pesquisa de grupos con riesgo suicida (Edelstein et al., 2009; Woods, Zimmerman, Carlin, Hill \& Kaslow, 2013).

Existen versiones de la RFL en español con validaciones en Colombia y Perú (Cassaretto \& Martínez, 2012; García Valencia et al., 2009). Estos estudios muestran que la RFL constituye un aporte, ya que muestra diferencias significativas en sus puntajes, entre individuos con y sin conductas e ideas suicidas (GarcíaValencia et al., 2007; Linehan et al., 1983). En la misma línea, existen motivos que inhiben la conducta suicida que podrían diferenciar y caracterizar consultantes de salud mental con distinta conducta suicida (Echávarri, Morales et al., 2015; García Valencia et al., 2009). Es así que la RFL se perfila como un instrumento que puede aportar en la evaluación más precisa del riesgo suicida. Cabe resaltar que esta escala identifica las razones que evitan que la persona tome la decisión de suicidarse y, por lo tanto, se enfoca en aspectos adaptativos y que vinculan a la vida (Linehan et al., 1983). La escala RFL constituye, entonces, un instrumento complementario a las mediciones de las características del intento de suicidio y de los factores de riesgo, aportando información útil para detectar los recursos y fortalezas. Así, también, sus resultados son aplicables en las intervenciones preventivas y terapéuticas (García Valencia et al., 2009).

Si bien existen versiones en español, resulta necesario contar con una validación para Chile que incluya las adaptaciones necesarias al contexto cultural y establecer las propiedades psicométricas y el puntaje de corte propios para la población chilena. En consecuencia, el presente estudio se propuso validar para Chile la escala RFL. Contar con esta escala validada y adaptada es un claro aporte, tanto por sus aplicaciones clínicas como para la investigación y, más aún, considerando que ya está siendo utilizada en investigaciones chilenas.

\section{Método}

La escala RFL indaga acerca de la importancia atribuida a las razones para vivir cuando se está pensando en suicidarse (Linehan et al., 1983). El estudio fue realizado en una población de consultantes a salud mental de la Región Metropolitana de Chile, con la autorización de la autora Dra. Marsha Linehan (Linehan et al., 1983). Se realizó una traducción al español de la versión original en inglés y se presentó a un panel de cuatro profesionales, compuesto por tres psicólogas y un psiquiatra. Se realizaron cambios mínimos de lenguaje y estilo, con el propósito de hacer las preguntas más comprensibles para la población chilena. Se tuvo en consideración mantener el significado exacto del instrumento original, para lo que se comparó el documento traducido con una nueva traducción al inglés. Esta fue revisada por una profesional anglo parlante nativa, quien contrastó la versión original con la versión traducida. Se efectuó una prueba piloto con los primeros 20 pacientes evaluados, mediante lo cual se probó la aplicabilidad de la prueba, evaluando la comprensión de las preguntas y del lenguaje utilizado. Se respetó la dirección de las preguntas del instrumento original y se verificó el tiempo requerido para su administración.

\section{Participantes}

Se obtuvo una muestra intencionada y consecutiva de 705 consultantes a salud mental, clasificada en dos grupos: (a) Grupo con conducta suicida, compuesto por consultantes por intento suicida e ideación suicida actual ( $n=347)$ y (b) Grupo sin conducta suicida, compuesto por consultantes sin intento ni ideación suicida actual $(n=358)$. Se consideró la ausencia de riesgo suicida actual no presentar ideación o intentos de suicidio al menos durante el último año. El promedio de edad de los participantes fue de 39,68 $\pm 14,86$ años, en un rango de 14 a 83 años, el 79,86\% $(n=563)$ eran mujeres, el 36,79\% $(n=259)$ se encontraba casado, el $41,76 \%$ $(n=294)$, soltero y el $47,13 \%(n=328)$ contaba con estudios superiores. Los participantes eran consultantes a servicios ambulatorios y de hospitalización, de tres centros asistenciales correspondientes a distintos niveles socioeconómicos de la Región Metropolitana de Santiago de Chile. Este estudio se realizó entre junio de 2010 y diciembre de 2014. La descripción clínica y sociodemográfica de los consultantes evaluados se puede observar en la Tabla 1. 
Tabla 1

Características Sociodemográficas de los Consultantes Evaluados

\begin{tabular}{|c|c|c|c|c|}
\hline Variable & $\begin{array}{c}\text { Total } \\
(N=705)\end{array}$ & $\begin{array}{c}\text { Grupo sin conducta } \\
\text { suicida (G0) } \\
(N=358)\end{array}$ & $\begin{array}{c}\text { Grupo con conducta } \\
\text { suicida (G1) } \\
(N=347)\end{array}$ & $\begin{array}{l}\text { Prueba de diferencia de } \\
\text { grupos (G0 vs G1) }\end{array}$ \\
\hline Edad en años $M(D E)$ & $39,68(14,86)$ & $42,12(14,46)$ & $37,16(14,86)$ & $\begin{array}{c}t(703)=4,49, p<0,001 \\
d=0,339 \\
95 \% \text { IC para diferencia } \\
{[-7,13,-2,79]}\end{array}$ \\
\hline $\begin{array}{c}\text { Género } N(\%) \\
\text { Femenino } \\
\text { Masculino }\end{array}$ & $\begin{array}{l}563(79,86) \\
142(20,14)\end{array}$ & $\begin{array}{r}287(80,17) \\
71(19,83)\end{array}$ & $\begin{array}{r}276(79,54) \\
71(20,46)\end{array}$ & $\begin{array}{c}\text { Test Exacto de Fisher }= \\
0,96, \\
p=0,851\end{array}$ \\
\hline $\begin{array}{l}\text { Estado civil } N(\%) \\
\text { Casado/a } \\
\text { Unión libre } \\
\text { Soltero/a } \\
\text { Viudo/a o separado/a }\end{array}$ & $\begin{array}{l}259(36,79) \\
32(4,55) \\
294(41,76) \\
119(16,90)\end{array}$ & $\begin{aligned} 148 & (41,46) \\
19 & (5,32) \\
126 & (32,29) \\
64 & (17,93)^{\mathrm{a}}\end{aligned}$ & $\begin{aligned} 111 & (31,99) \\
13 & (3,75) \\
168 & (48,15) \\
55 & (15,85)\end{aligned}$ & $\begin{aligned} \chi^{2}(3, N= & 704)=12,95, p \\
& =0,004\end{aligned}$ \\
\hline Tiene hijos $N(\%)$ & $454(64,40)$ & $249(69,55)$ & $205(59,07)$ & $\begin{array}{c}\text { Test Exacto de Fisher }= \\
1,58 \\
p=0,004\end{array}$ \\
\hline $\begin{array}{l}\text { Escolaridad } N(\%) \\
\text { Con estudios superiores } \\
\text { Sin estudios superiores }\end{array}$ & $\begin{array}{l}328(47,13) \\
368(52,87)\end{array}$ & $\begin{array}{l}154(43,26) \\
202(56,74)^{\mathrm{b}}\end{array}$ & $\begin{array}{l}174(51,18) \\
166(48,82)^{\mathrm{c}}\end{array}$ & $\begin{array}{c}\text { Test Exacto de Fisher }= \\
0,72, \\
p=0,040\end{array}$ \\
\hline $\begin{array}{l}\text { Ocupación } N(\%) \\
\text { Trabajo remunerado } \\
\text { Estudiante } \\
\text { Desempleado } \\
\text { Dueña de casa } \\
\text { Sin actividad }\end{array}$ & $\begin{array}{c}364(52,07) \\
152(21,75) \\
41(5,87) \\
129(18,46) \\
13(1,86)\end{array}$ & $\begin{array}{c}215(60,73) \\
54(15,25) \\
19(5,37) \\
59(16,67) \\
7(1,98)^{\mathrm{d}}\end{array}$ & $\begin{aligned} 149 & (43,19) \\
98 & (28,41) \\
22 & (6,4) \\
70 & (20,29) \\
6 & (1,74)^{\mathrm{e}}\end{aligned}$ & $\begin{array}{c}\chi^{2}(4, N=699)=25,83 \\
p<0,001\end{array}$ \\
\hline
\end{tabular}

Nota. $\mathrm{a}=1$ caso $\sin$ respuesta $; \mathrm{b}=2$ casos $\sin$ respuesta; $\mathrm{c}=7$ casos no entregaron información; $\mathrm{d}=4$ casos no entregaron información; e $=2$ casos no entregaron información.

\section{Instrumentos}

Escala Reasons for Living (RFL; Linehan et al., 1983). Fue traducida al español en este estudio. La escala evalúa las razones disuasivas de intentar suicidarse, por medio de 48 ítems puntuados mediante una escala Likert de 6 puntos (ver la escala en el Anexo), desde 1 (para nada importante) a 6 (extremadamente importante). Así, cada ítem es una afirmación que el evaluado puntúa según la importancia que le atribuye como motivo para no suicidarse. Los ítems se ordenan en seis dimensiones, que reflejan la importancia que el mismo paciente otorga a distinto tipo de motivos: (a) Supervivencia y afrontamiento (SUPAF), (b) Miedo a la desaprobación social (MDESS), (c) Responsabilidad con familia (RESFAM), (d) Preocupación por hijos (PREOC), (e) Miedo al suicidio (MSUIC) y (f) Objeciones morales (OBMOR). Los ítems de cada dimensión pueden observarse en la Tabla 5. El rango teórico total de cada dimensión es desde 1 a 6 , correspondiendo un mayor puntaje a una mayor importancia otorgada a la misma. El punto de corte (gold standard) de la versión original del instrumento es de 3,8 puntos, bajo el cual se considera la presencia de riesgo suicida (Linehan et al., 1983).

Outcome Questionnaire (OQ-45.2) en su versión validada para la población chilena (Lambert et al., 1996; von Bergen \& de la Parra, 2002). Es una escala de tipo Likert de 45 ítems, que evalúa cómo se ha sentido la persona en los últimos días en cuanto a: (a) La presencia de sintomatología ansiosa y depresiva (25 ítems), por ejemplo, "Me siento inútil"; (b) La calidad de las relaciones interpersonales (11 ítems), como, por ejemplo, "Me siento solo" y (c) El sentimiento de adecuación en el rol social (en la familia, empleo y ocio; 9 ítems), como, por ejemplo, "Tengo problemas en el trabajo". Ante cada afirmación se marca una de las siguientes alternativas: nunca (0), casi nunca (1), a veces (2), con frecuencia (3) o casi siempre (4). El rango del puntaje del total del $\mathrm{OQ}$ es de 0 a 180 puntos, indicando un puntaje mayor un mayor disconfort.

El alfa de Cronbach de la validación chilena fue de $\alpha=0,93$ para la escala total, $\alpha=0,91$ para la subescala Sintomatología ansiosa y depresiva, $\alpha=0,74$ para la subescala Bienestar/malestar en las relaciones 
interpersonales y $\alpha=0,71$ para la subescala Sentimientos de adecuación en el rol social. Para la muestra del presente estudio se observó un alfa de Cronbach de $\alpha=0,93$ para la escala total.

APGAR Familiar en su versión validada al español (Maddaleno, Horwitz, Jara, Florenzano \& Zalazar, 1987; Smilkstein, 1978). Es una escala que mide la satisfacción respecto del funcionamiento familiar. Consta de cinco preguntas con respuestas de tipo Likert, con las alternativas de nunca (0), algunas veces (1) y siempre (2). Se evalúan de manera general cinco aspectos de la vida familiar: (a) Adaptabilidad, (b) Participación, (c) Gradiente de crecimiento, (d) Afecto y (e) Resolución. Los ítems son del tipo "Me satisface la ayuda que recibo de mi familia". El puntaje total oscila entre 0 y 10 puntos, representando un mayor puntaje una mayor satisfacción con el funcionamiento familiar. El alfa de Cronbach de la validación del instrumento para Chile fue de $\alpha=0,87,95 \%$ IC $[0,85,0,88]$.

State Trait Anger Expression Inventory (STAXI; Forgays, Forgays \& Spielberger, 1997) en su versión validada al español (Leibovich de Figueroa, Schmidt \& Gol, 2001). Es una escala de 44 afirmaciones con respuestas de tipo Likert, que evalúa, desde la perspectiva del paciente, la experiencia y expresión de rabia, en dos dimensiones (a) Estado de rabia (10 ítems), que corresponde a la intensidad de los sentimientos de enojo en un momento determinado, con afirmaciones como "Me siento como que quisiera golpear a alguien" y (b) Rasgo de rabia (10 ítems), que es la disposición para experimentar enojo, con afirmaciones como "Tengo un temperamento explosivo". El instrumento distingue, además, la expresión de la rabia en tres direcciones: (a) Enojo hacia afuera, que se refiere a la exteriorización de este dirigido a otras personas u objetos, con afirmaciones como "Hago cosas como dar portazos". Se la considera una forma inadecuada de expresar el enojo; (b) Enojo hacia adentro, que es la supresión de los sentimientos de enojo, con afirmaciones como "Hiervo por dentro pero no lo demuestro". Esta dirección que toman dichos sentimientos también se la considera inadecuada; y (c) Control de enojo, que se define como el intento de manejar la expresión del mismo, pudiendo expresarlo adecuadamente, con afirmaciones del tipo "Si alguien me molesta estoy preparado para decirle cómo me siento". El rango de respuestas es En total desacuerdo=1, Medianamente de acuerdo $=2$ y Muy de acuerdo $=3$, siendo los puntajes más altos los que indican mayor disfuncionalidad en el estado y rasgo. Los puntajes se calculan del siguiente modo: Estado de rabia (evaluado en preguntas 1-10) con rango teórico entre 10-30; Rasgo de rabia (evaluado en preguntas 11-20) con rango teórico entre 10-30 y La expresión del enojo (rango teórico 16-48), que se compone de la suma de la Exteriorización (evaluada en preguntas 22, 27, 29, 32, 34, 39, 42 y 43) con rango teórico entre 8-24, y el Guardado del enojo (evaluado en preguntas 23, 25, 26, 30, 33, 36, 37 y 41) con rango entre 8-24, a lo que se le resta el Control del enojo (evaluado en preguntas 21, 24, 28, 31, 35, 38, 40 y 44) con rango teórico entre 8-24. Un mayor puntaje es indicador de mayor disfuncionalidad en la expresión de la rabia.

El alfa de Cronbach para la muestra estudiada fue de $\alpha=0,91$ para la escala total, $\alpha=0,88$ para la subescala Estado de rabia, $\alpha=0,81$ para la subescala Rasgo de rabia, $\alpha=0,84$ para la subescala Control de la rabia, $\alpha=0,61$ para la subescala Guardado de la rabia, $\alpha=0,65$ para la subescala Externalización de la rabia y a = 0,52 para la subescala Expresión de la rabia.

Depressive Experiences Questionnaire en su versión en español (DEQ; Blatt, D’Afflitti \& Quinlan, 1976) en su versión adaptada al español (Gargurevich, Luyten \& Corveleyn, 2008). Es una escala de 66 preguntas, con respuesta de tipo Likert (con Es una escala de 66 preguntas, con respuesta de tipo Likert (con puntajes que van de 1 En total desacuerdo a 7 En total acuerdo), que mide dos factores relacionados con dos formas de vivencia depresiva: (a) Autocrítica y (b) Dependencia. Existe un tercer factor, Autoeficacia, considerado protector del riesgo suicida (Blatt et al., 1976). Los puntajes se obtienen factorialmente, en los que cada una de las preguntas aporta, en alguna magnitud, a cada uno de los factores.

$\mathrm{El}$ alfa de Cronbach para la muestra estudiada fue de $\alpha=0,60$ para la subescala Dependencia, $\alpha=0,79$ para la subescala Autocrítica y $\alpha=0,69$ para la subescala Autoeficacia. Tomadas en conjunto, las tres subescalas presentan un $\alpha=0,84$.

La utilización de estas cuatro escalas, adicionales a la RFL, se relacionó con la posibilidad de determinar la validez de constructo del instrumento, ya que en casos como el suicidio se espera la acción concurrente y asociada de distintas variables. La selección de instrumentos que miden síntomas, manejo de la agresión, percepción del cuidado parental y estilo de vivencia depresiva se debe a que estudios cualitativos sugieren su relevancia en la conducta suicida (Morales et al., 2015). 


\section{Procedimiento}

En colaboración con los equipos tratantes se determinaron los diagnósticos psiquiátricos, se solicitó a los consultantes su participación voluntaria y la firma de consentimiento/asentimiento informado. El estudio fue llevado a cabo siguiendo los criterios de los respectivos Comités de Ética de la Pontificia Universidad Católica de Chile y del Hospital Sótero del Río, de manera que los participantes involucrados fueron informados y participaron voluntariamente, de acuerdo a los criterios estipulados por la declaración de Helsinki (Manzini, 2000). En los casos de personas menores de edad, firmaron su consentimiento informado los padres o tutores legales. Además, el menor de edad firmó su asentimiento informado. Se recopiló información respecto del motivo de consulta y las características sociodemográficas de los participantes.

Los criterios de inclusión fueron: consultantes a salud mental, mayores de 14 años, de ambos sexos, con juicio de realidad conservado, y en condiciones cognitivas y emocionales de responder a las preguntas de los instrumentos de evaluación. Fueron excluidos consultantes según los siguientes criterios: presentar diagnósticos de trastorno por dependencia al alcohol o sustancias, trastorno psicótico, trastorno cognitivo y trastorno alimentario. La decisión de excluir estas patologías respondió al propósito de enfocar el estudio en los trastornos del ánimo. Asimismo, en esos casos la consulta estaba motivada por razones relacionadas con sus diagnósticos principales y no con el riesgo suicida.

Los instrumentos de evaluación fueron de auto reporte. No obstante, en todos los casos las evaluadoras acompañaron a los participantes en el proceso de responder a las preguntas, deteniéndose sobre ellas, en caso de que presentaran dificultad para comprender. Se aplicaron todos los instrumentos a todos los participantes en el siguiente orden: OQ, APGAR Familiar, STAXI, DEQ y RFL.

\section{Análisis de Datos}

El análisis estadístico fue efectuado con el programa R (R Core Team, 2016), incluyendo los siguientes procedimientos:

1. Análisis descriptivo de las características sociodemográficas y clínicas de los consultantes evaluados, determinando la existencia de posibles diferencias entre los grupos usando pruebas $t$ de Student para muestras independientes en los casos de las variables cuantitativas. En el caso de variables categóricas, se utilizó $\chi^{2}$ para aquellas variables con más de dos categorías y el test exacto de Fischer en el caso de variables con dos categorías. Si bien hubo algunos casos sin respuesta (ver Tabla 1), ellos no sobrepasaron el $2 \%$, por lo tanto, no fueron imputados.

2. Análisis de consistencia interna de los instrumentos utilizados, mediante alfa de Cronbach. Este se calculó en base a un mínimo de 680 casos que respondieron todas las preguntas del set.

3. Análisis de validez de constructo, para el cual se trabajó con los 680 casos señalados. Se analizó la correlación lineal de Pearson entre las subescalas originales de la RFL, esperando una correlación directa entre las dimensiones, y se testeó la estructura teórica propuesta en el instrumento original, por medio de un análisis factorial exploratorio, en base al modelo de máxima verosimilitud con rotación varimax. La adecuación de los datos para el análisis factorial fue testeada con la medida de adecuación muestral de Kaiser-Meyer-Olkin (KMO) y la prueba de esfericidad de Bartlett. Para la verificación de la estructura teóricamente propuesta, se efectuó un análisis factorial confirmatorio, el que fue aplicado a los 643 casos adultos que contestaron a todas las preguntas. Para evaluar el ajuste del modelo se utilizaron $\chi^{2 / g l}$, el índice comparativo de Bentler-Bonett (CFI), el índice de Tucker Lewis (TLI), el error cuadrático medio de aproximación (RMSEA) y la raíz del residuo cuadrático medio estandarizado (SRMR). Se consideran adecuados valores de CFI y TLI superiores o iguales a 0,95, valores RMSEA menores o iguales a 0,05 y valores SRMR inferiores o iguales a 0,08 (Kline, 2011).

4. Análisis de validez concurrente, para el cual se trabajó con el total de casos que contaban con información completa tanto en la RFL como en las otras escalas, con $n$ que fluctuó entre 633 y 675 . La convergencia con otros instrumentos se evaluó calculando las correlaciones lineales de Pearson entre los puntajes de las subescalas de la RFL y los de otras escalas clínicas asociadas a riesgo suicida: OQ, APGAR Familiar, STAXI y DEQ.

5. Determinación de un punto de corte para la detección de riesgo suicida, mediante un análisis de curva ROC, utilizando la librería pROC (Robin et al., 2011). Esto permitió identificar el punto de corte que equilibra la sensibilidad y especificidad del puntaje de la RFL, que diferencia entre grupo con y sin riesgo 
suicida. Este permite optimizar la precisión de tamizaje en relación con la presencia de un riesgo suicida, comparando como grupo control a los sujetos sin ideación ni intento suicida y como grupo de riesgo a los sujetos que pertenecen al grupo con ideación o intento suicida. Cabe mencionar que no existe un estándar absoluto para la interpretación del área bajo la curva (AUC por su sigla en inglés): es aceptado que un AUC igual a 0,5 representa un test sin valor predictivo, interpretándose valores entre 0,6 y 0,7 como aceptables, entre 0,7 a 0,9 como buenos y sobre 0,9 como excelentes (Robin et al., 2011).

\section{Resultados}

En cuanto a las características sociodemográficas ya presentadas (Tabla 1), solo en género no se observó diferencia entre los grupos con y sin conducta suicida. El grupo con conducta suicida es un grupo más joven (Tabla 2), con una menor proporción de personas con trabajo remunerado y mayor proporción de estudiantes, más solteros y sin hijos y un mayor porcentaje de personas con estudios superiores (ver Tabla 1). Desde el punto de vista diagnóstico, el grupo con conducta suicida presenta una mayor proporción de trastorno depresivo mayor, mientras en el grupo sin conducta suicida se observan más casos de trastornos de ansiedad (Tabla 3).

Tabla 2

Distribución por Edad, Según Grupo

\begin{tabular}{lcccc}
\hline Edad & Total & Grupo sin \\
$N(\%)$ & $\begin{array}{c}\text { Grupo con } \\
\text { conducta suicida } \\
N(\%)\end{array}$ & $\begin{array}{c}\text { conducta suicida } \\
N(\%)\end{array}$ & $\chi^{2}$ \\
\hline $14-19$ años & $80(11,36)$ & $25(6,98)$ & $55(15,90)$ & \\
$20-29$ años & $129(18,32)$ & $57(15,92)$ & $72(20,81)$ & \\
$30-39$ años & $135(19,18)$ & $66(18,44)$ & $69(19,94)$ & $\chi^{2}(5 ; N=714)=28,98$, \\
$40-49$ años & $141(20,03)$ & $85(23,74)$ & $56(16,19)$ & $p<0,001$ \\
$50-59$ años & $166(22,16)$ & $81(22,63)$ & $65(21,67)$ & \\
60 años y más & $63(8,95)$ & $44(12,29)$ & $19(5,49)$ & \\
\hline
\end{tabular}

Tabla 3

Distribución Según Diagnóstico de los Consultantes Evaluados

\begin{tabular}{lcccc}
\hline \multicolumn{1}{c}{ Dotal } & Grupo sin & Grupo con & \\
& $(N=685)$ & $\begin{array}{c}\text { conducta suicida } \\
(N=340)\end{array}$ & $\begin{array}{c}\text { conducta suicida } \\
(N=345)\end{array}$ & $\chi^{2}$ \\
& $N(\%)$ & $N(\%)$ & $N(\%)$ & \\
\hline Trastorno adaptatico & $70(10,22)$ & $44(12,94)$ & $26(7,54)$ & \\
Trastorno por ansiedad & $65(9,49)$ & $49(14,41)$ & $16(4,64)$ & \\
Distimia & $11(1,60)$ & $6(1,77)$ & $5(1,45)$ & \\
Depresión leve & $26(3,79)$ & $17(5,00)$ & $9(2,61)$ & $\chi^{2}(8 ; N=685)=65,19$, \\
Depresión moderada & $53(7,74)$ & $29(8,53)$ & $24(6,96)$ & \\
Depresión mayor & $301(43,94)$ & $100(29,41)$ & $201(68,26)$ & \\
Trastorno bipolar & $110(16,06)$ & $66(19,41)$ & $44(12,75)$ & \\
Trastorno mixto & $16(2,33)$ & $11(3,24)$ & $5(1,45)$ & \\
Otro & $33(4,82)$ & $18(5,29)$ & $15(4,35)$ & \\
\hline
\end{tabular}

En la Tabla 4 se presentan los resultados del análisis de consistencia interna de las subescalas de la RFL en la muestra estudiada. Como se observa, la consistencia interna de la escala y de la subescala Supervivencia y afrontamiento es alta; en cambio, en Miedo al suicidio y Miedo a la desaprobación social fue más baja. 

SZMULEWICZ Y TOMICIC

Tabla 4

Consistencia Interna de la Escala y Subescalas de la RFL $(N=680)$

\begin{tabular}{lccc}
\hline \multicolumn{1}{c}{ Subescalas y escala } & $\begin{array}{c}\text { Número de } \\
\text { ítems }\end{array}$ & $\begin{array}{c}\text { Alfa de } \\
\text { Cronbach }\end{array}$ & $\begin{array}{c}\text { Media de } \\
\text { correlaciones } \\
\text { inter ítem }\end{array}$ \\
\hline Supervivencia y afrontamiento & 24 & $0,96[0,95,0,96]$ & 0,49 \\
Miedo a la desaprobación social & 3 & $0,75[0,72,0,79]$ & 0,51 \\
Responsabilidad con la familia & 7 & $0,82[0,80,0,84]$ & 0,41 \\
Preocupación por los hijos & 3 & $0,87[0,85,0,88]$ & 0,69 \\
Miedo al suicidio & 7 & $0,72[0,69,0,76]$ & 0,27 \\
Objeciones morales & 4 & $0,77[0,74,0,80]$ & 0,42 \\
Escala total & 48 & $0,95[0,94,0,95]$ & 0,29 \\
\hline
\end{tabular}

Nota. Los números entre corchetes indican el intervalo de confianza a un $95 \%$ de seguridad de la consistencia interna calculada.

Los análisis para la muestra adulta mostraron buenos niveles de ajuste para la realización del análisis factorial, $\mathrm{KMO}=0,95$ y $\chi^{2}$ Bartlett $(1128, N=603)=17762,44, p<0,001$. El análisis detectó una solución de cinco factores con eigenvalues superiores a 1, no logrando diferenciar las subescalas de Miedo al suicidio y Miedo a la desaprobación social, las que aparecen fusionadas en un solo factor. Las cargas factoriales obtenidas se pueden apreciar en la Tabla 5.

Tabla 5

Análisis Factorial de los Puntajes de los 48 Ítems de la Escala RFL: Adultos (18-65 Años)

\begin{tabular}{|c|c|c|c|c|c|c|c|}
\hline Ítem & $\mathrm{h}_{2}$ & 1 & 2 & 3 & 4 & 5 & Subescala \\
\hline 24. Siento amor por la vida & 0,805 & 0,857 & & & & & SUPAF \\
\hline 19. Me quiero lo suficiente como para vivir & 0,729 & 0,804 & & & & & SUPAF \\
\hline 36. Tengo la valentía de enfrentar la vida & 0,677 & 0,800 & & & & & SUPAF \\
\hline 20. La vida es demasiado bonita y valiosa como para terminarla & 0,731 & 0,798 & & & & & SUPAF \\
\hline 42. Tengo la motivación interior para sobrevivir & 0,660 & 0,789 & & & & & SUPAF \\
\hline 4. Tengo deseos de vivir & 0,614 & 0,765 & & & & & SUPAF \\
\hline 37. Estoy feliz y contento/a con mi vida & 0,584 & 0,737 & & & & & SUPAF \\
\hline $\begin{array}{l}\text { 40. Tengo la esperanza de que las cosas mejorarán y que el futuro será más } \\
\text { feliz }\end{array}$ & 0,643 & 0,728 & & & & & SUPAF \\
\hline 13. Tengo planes futuros que deseo llevar a cabo & 0,583 & 0,722 & & & & & SUPAF \\
\hline 35. Todavía tengo muchas cosas por hacer & 0,624 & 0,721 & & & & & SUPAF \\
\hline $\begin{array}{l}\text { 17. Quiero vivir toda experiencia que la vida me ofrezca y hay muchas } \\
\text { experiencias que no he tenido aún }\end{array}$ & 0,608 & 0,720 & & & & & SUPAF \\
\hline 22. Creo que puedo encontrar otra solución a mis problemas & 0,601 & 0,710 & & & & & SUPAF \\
\hline 14. No importa cuán mal me sienta, sé que no durará para siempre & 0,549 & 0,706 & & & & & SUPAF \\
\hline 10. No quiero morir & 0,487 & 0,663 & & & & & SUPAF \\
\hline 44. Creo que puedo encontrar un propósito en mi vida, una razón para vivir & 0,495 & 0,633 & & & & & SUPAF \\
\hline 12. La vida es todo lo que tenemos y es mejor que nada & 0,573 & 0,633 & & & & & SUPAF \\
\hline 45. No veo razón para apurar la muerte & 0,462 & 0,592 & & & & & SUPAF \\
\hline 25. Soy demasiado estable para matarme & 0,467 & 0.587 & & & & & SUPAF \\
\hline 32. Creo que todo puede resolverse para mejor & 0,417 & 0,570 & & & & & SUPAF \\
\hline 2. Creo que puedo aprender a ajustarme a sobrellevar los problemas & 0,373 & 0,562 & & & & & SUPAF \\
\hline 16. Quiero y disfruto mucho a mi familia, no quisiera dejarlos & 0,538 & 0,503 & & 0,442 & & & RESFAM \\
\hline 3. Creo que tengo control sobre mi vida y mi destino & 0,258 & 0,495 & & & & & SUPAF \\
\hline 29. Siento curiosidad acerca de lo que ocurrirá en el futuro & 0,400 & 0,444 & & & & & SUPAF \\
\hline 39. Creo que suicidarme no lograría ni solucionaría nada & 0,372 & 0,435 & & & & & SUPAF \\
\hline $\begin{array}{l}\text { 8. No creo que las cosas se pongan tan mal o desesperanzadoras como para } \\
\text { preferir estar muerto/a }\end{array}$ & 0,230 & 0,417 & & & & & SUPAF \\
\hline
\end{tabular}




\begin{tabular}{|c|c|c|c|c|c|c|c|}
\hline Ítem & $\mathrm{h}_{2}$ & 1 & 2 & 3 & 4 & 5 & Subescala \\
\hline 41. Otras personas pensarían que soy débil o egoísta & 0,485 & & 0,649 & & & & MDESS \\
\hline 43. No quisiera que otros pensaran que no tuve control sobre mi vida & 0,452 & & 0,619 & & & & MDESS \\
\hline 46. Soy tan inepto/a que mi método no funcionaría & 0,397 & & 0,604 & & & & MSUIC \\
\hline 26. Soy cobarde y no tengo las agallas para hacerlo & 0,345 & & 0,567 & & & & MSUIC \\
\hline 31. Me preocupa lo que los demás pensarían de mí & 0,339 & & 0,541 & & & & MDESS \\
\hline 38. Me da miedo el acto mismo de suicidarme (el dolor, la sangre y la violencia) & 0,396 & & 0,534 & & & & MSUIC \\
\hline 33. No podría decidir dónde, cuándo y cómo hacerlo & 0,259 & & 0,495 & & & & MSUIC \\
\hline 15. Tengo miedo a lo desconocido & 0,258 & & 0,464 & & & & MSUIC \\
\hline 34. Lo considero moralmente malo & 0,442 & & 0,427 & & & & OBMOR \\
\hline 18. Tengo miedo a que el método que elija para suicidarme falle & 0,280 & & 0,405 & & & & MSUIC \\
\hline 6. Tengo miedo a la muerte & 0,193 & & 0,293 & & & & MSUIC \\
\hline 47. No quisiera que mi familia se sintiera culpable después & 0,776 & & & 0,809 & & & RESFAM \\
\hline 48. No quisiera que mi familia piense que soy egoísta y cobarde & 0,657 & & & 0,712 & & & RESFAM \\
\hline 30. Le dolería demasiado a mi familia y no quiero que sufran & 0,554 & & & 0,594 & & & RESFAM \\
\hline 1. Tengo una responsabilidad y estoy comprometido/a con mi familia & 0,433 & & & 0,426 & & & RESFAM \\
\hline 9. Mi familia depende de mí y me necesita & 0,402 & & & 0,389 & & & RESFAM \\
\hline 7. Mi familia podría creer que no los quise & 0,153 & & & 0,289 & & & RESFAM \\
\hline 21. No sería justo dejar a mis niños/as para que otros/as los/as cuiden & 0,910 & & & & 0,877 & & PREOC \\
\hline 28. El efecto en mis hijos podría ser dañino & 0,616 & & & & 0,716 & & PREOC \\
\hline 11. Quiero ver crecer a mis hijos/as & 0,656 & & & & 0,687 & & PREOC \\
\hline 5. Pienso que solo Dios tiene derecho a terminar con una vida & 0,636 & & & & & 0,670 & OBMOR \\
\hline 27. Mis creencias religiosas lo prohíben & 0,527 & & & & & 0,641 & OBMOR \\
\hline 23. Tengo miedo ir al infierno & 0,443 & & & & & 0,470 & OBMOR \\
\hline$\%$ varianza explicada & & 25,92 & 8,69 & 5,70 & 4,96 & 3,80 & \\
\hline Eigenvalue & & 12,44 & 4,17 & 2,73 & 2,38 & 1,83 & \\
\hline
\end{tabular}

Nota. $N=603$ (muestra entre 18 y 65 años). Método de máxima verosimilitud con rotación varimax. Se reportan factores con eigenvalue $>1$. En cada ítem se muestran cargas factoriales sobre 0,40 , salvo en los casos en que el ítem tenga cargas factoriales inferiores a 0,40 en todos los factores. En ese caso, se reporta la máxima carga detectada. La columna "Subescala" indica la pertenencia de cada ítem a la escala original de la RFL, usando la siguientes siglas: SUPAF $=$ Supervivencia y afrontamiento, MDESS $=$ Miedo a la desaprobación social, RESFAM $=$ Responsabilidad con la familia, PREOC $=$ Preocupación por los hijos, MSUIC = Miedo al suicidio, OBMOR = Objeciones morales.

El análisis confirmatorio de los factores de las subescalas originales no logró obtener ajustes aceptables: $\chi^{2} / g l=3,368, \mathrm{CFI}=0,844, \mathrm{TLI}=0,835, \mathrm{RMSEA}=0,063,90 \%$ IC RMSEA [0,060, 0,065], SRMR $=0,081$. De esta forma, el comportamiento estadístico del test no estaría confirmando la distinción entre seis subescalas, tal como fue la propuesta inicial de sus autores. En consideración de la alta correlación detectada entre la subescala Supervivencia y afrontamiento con el puntaje total del test (ver Tabla 6), se hizo un nuevo análisis factorial confirmatorio, incluyendo solamente los ítems que corresponden a la subescala de Supervivencia y afrontamiento. Esta propuesta de un modelo unidimensional sí logra niveles de ajuste adecuados: $\chi^{2} / g l=2,093, \mathrm{CFI}=0,980, \mathrm{TLI}=0,974, \mathrm{RMSEA}=0,042,90 \%$ IC RMSEA [0,036, 0,048], $\mathrm{SRMR}=0,026$.

Desde otro punto de vista, la estructura teórica propuesta apunta a distintas áreas temáticas asociadas a las razones para vivir, de modo que encontrar correlaciones directas entre los puntajes escalares podría ser considerado como una evidencia de la validez de constructo y como un argumento para la mantención de la distinción conceptual de las subescalas. El resultado del análisis de correlación inter escalas del instrumento RFL, calculadas en base a lo definido en el test original y que confirma lo recién expuesto, puede verse en la Tabla 6.

Respecto de la validez concurrente de la RFL, el detalle de las correlaciones entre sus subescalas y los otros instrumentos empleados para medir variables clínicas asociadas a riesgo suicida se puede observar en la Tabla 7. 
Tabla 6

Correlaciones Lineales de Pearson entre las Subescalas de la RFL

\begin{tabular}{|c|c|c|c|c|c|c|}
\hline & MDESS & RESFAM & PREOC & MSUIC & OBMOR & RFL \\
\hline (SUPAF) Supervivencia y afrontamiento & $\begin{array}{c}0,35^{*} \\
(n=672)\end{array}$ & $\begin{array}{c}0,57^{*} \\
(n=672)\end{array}$ & $\begin{array}{c}0,50^{*} \\
(n=668)\end{array}$ & $\begin{array}{c}0,22^{*} \\
(n=671)\end{array}$ & $\begin{array}{c}0,44^{*} \\
(n=672)\end{array}$ & $\begin{array}{c}0,91^{*} \\
(n=664)\end{array}$ \\
\hline (MDESS) Miedo desaprobación social & & $\begin{array}{c}0,38^{*} \\
(n=678)\end{array}$ & $\begin{array}{c}0,20^{*} \\
(n=673)\end{array}$ & $\begin{array}{c}0,53^{*} \\
(n=675)\end{array}$ & $\begin{array}{c}0,41^{*} \\
(n=678)\end{array}$ & $\begin{array}{c}0,56^{*} \\
(n=664)\end{array}$ \\
\hline (RESFAM) Responsabilidad con familia & & & $\begin{array}{c}0,47^{*} \\
(n=679)\end{array}$ & $\begin{array}{c}0,32^{*} \\
(n=673)\end{array}$ & $\begin{array}{c}0,40^{*} \\
(n=675)\end{array}$ & $\begin{array}{c}0,73^{*} \\
(n=664)\end{array}$ \\
\hline (PREOC) Preocupación por hijos & & & & $\begin{array}{c}0,17^{*} \\
(n=671)\end{array}$ & $\begin{array}{c}0,33^{*} \\
(n=673)\end{array}$ & $\begin{array}{c}0,59^{*} \\
(n=664)\end{array}$ \\
\hline (MSUIC) Miedo al suicidio & & & & & $\begin{array}{c}0,44^{*} \\
(n=679)\end{array}$ & $\begin{array}{c}0,51^{*} \\
(n=664)\end{array}$ \\
\hline (OBMOR) Objeciones morales & & & & & & $\begin{array}{c}0,64^{*} \\
(n=664)\end{array}$ \\
\hline
\end{tabular}

$* p<0,001$

Tabla 7

Correlaciones de Puntajes de la Escala y Subescalas de la RFL con Otras Escalas y Subescalas Clínicas

\begin{tabular}{|c|c|c|c|c|c|c|c|c|}
\hline \multirow{2}{*}{ Escala } & \multirow{2}{*}{ Subescala } & \multicolumn{6}{|c|}{ Subescala de la RFL } & \multirow{2}{*}{ RFL } \\
\hline & & SUPAF & MDESS & RESFAM & PREOC & MSUIC & OBMOR & \\
\hline \multirow[t]{4}{*}{ OQ-45.2 } & $\begin{array}{l}\text { Sintomatología ansiosa y } \\
\text { depresiva }\end{array}$ & $\begin{array}{l}-0,53^{* * *} \\
(n=642)\end{array}$ & $\begin{array}{l}0,01 \\
(n=648)\end{array}$ & $\begin{array}{l}-0,21^{* * * *} \\
(n=647)\end{array}$ & $\begin{array}{l}-0,20^{* * * *} \\
(n=643)\end{array}$ & $\begin{array}{l}0,14^{*} \\
(n=644)\end{array}$ & $\begin{array}{l}-0,04 \\
(n=647)\end{array}$ & $\begin{array}{l}-0,37^{* * * *} \\
(n=633)\end{array}$ \\
\hline & $\begin{array}{l}\text { Calidad de relaciones } \\
\text { interpersonales }\end{array}$ & $\begin{array}{l}-0,46^{* * *} \\
(n=643)\end{array}$ & $\begin{array}{l}-0,05 \\
(n=649)\end{array}$ & $\begin{array}{l}-0,30 * * * \\
(n=648)\end{array}$ & $\begin{array}{l}-0,27 * * * \\
(n=644)\end{array}$ & $\begin{array}{l}0,06 \\
(n=645)\end{array}$ & $\begin{array}{l}-0,09 \\
(n=648)\end{array}$ & $\begin{array}{l}-0,37^{* * * *} \\
(n=634)\end{array}$ \\
\hline & Adecuación en rol social & $\begin{array}{l}-0,44^{* * *} \\
(n=642)\end{array}$ & $\begin{array}{l}-0,04 \\
(n=648)\end{array}$ & $\begin{array}{l}-0,19 * * * \\
(n=647)\end{array}$ & $\begin{array}{l}-0,15^{* *} \\
(n=643)\end{array}$ & $\begin{array}{l}-0,06 \\
(n=644)\end{array}$ & $\begin{array}{l}-0,08 \\
(n=647)\end{array}$ & $\begin{array}{l}-0,33^{* * *} \\
(n=633)\end{array}$ \\
\hline & Escala total & $\begin{array}{l}-0,55^{* * *} \\
(n=642)\end{array}$ & $\begin{array}{l}-0,01 \\
(n=648)\end{array}$ & $\begin{array}{l}-0,25^{* * *} \\
(n=673)\end{array}$ & $\begin{array}{l}-0,22^{* * *} \\
(n=643)\end{array}$ & $\begin{array}{l}-0,12 \\
(n=644)\end{array}$ & $\begin{array}{l}-0,06 \\
(n=647)\end{array}$ & $\begin{array}{l}-0,39 * * * \\
(n=633)\end{array}$ \\
\hline APGAR Familiar & Satisfacción familiar total & $\begin{array}{l}0,31^{* * *} \\
(n=667)\end{array}$ & $\begin{array}{l}0,06 \\
(n=674)\end{array}$ & $\begin{array}{l}0,40^{* * *} \\
(n=674)\end{array}$ & $\begin{array}{l}0,23^{* * * *} \\
(n=668)\end{array}$ & $\begin{array}{l}-0,01 \\
(n=670)\end{array}$ & $\begin{array}{l}0,12 \\
(n=673)\end{array}$ & $\begin{array}{c}0,32^{* * *} \\
(n=658)\end{array}$ \\
\hline \multirow[t]{6}{*}{ STAXI } & Estado de rabia & $\begin{array}{l}-0,30 * * * \\
(n=668)\end{array}$ & $\begin{array}{l}0,01 \\
(n=675)\end{array}$ & $\begin{array}{l}-0,15^{* *} \\
(n=675)\end{array}$ & $\begin{array}{l}-0,11^{* *} \\
(n=669)\end{array}$ & $\begin{array}{l}0,02 \\
(n=671)\end{array}$ & $\begin{array}{l}-0,06 \\
(n=674)\end{array}$ & $\begin{array}{l}-0,22^{* * *} \\
(n=659)\end{array}$ \\
\hline & Rasgo de rabia & $\begin{array}{l}-0,19 * * * \\
(n=669)\end{array}$ & $\begin{array}{l}0,13^{*} \\
(n=676)\end{array}$ & $\begin{array}{l}-0,02 \\
(n=675)\end{array}$ & $\begin{array}{l}-0,09 \\
(n=670)\end{array}$ & $\begin{array}{c}0,16^{* *} \\
(n=672)\end{array}$ & $\begin{array}{l}-0,03 \\
(n=675)\end{array}$ & $\begin{array}{l}-0,09 \\
(n=660)\end{array}$ \\
\hline & Guardado de rabia & $\begin{array}{l}-0,31^{* * *} \\
(n=669)\end{array}$ & $\begin{array}{l}0,01 \\
(n=676)\end{array}$ & $\begin{array}{l}-0,11 \\
(n=675)\end{array}$ & $\begin{array}{l}-0,14^{* *} \\
(n=670)\end{array}$ & $\begin{array}{l}0,11 \\
(n=672)\end{array}$ & $\begin{array}{l}-0,07 \\
(n=675)\end{array}$ & $\begin{array}{l}-0,21^{* * *} \\
(n=660)\end{array}$ \\
\hline & Externalización de rabia & $\begin{array}{l}0,01 \\
(n=669)\end{array}$ & $\begin{array}{l}0,06 \\
(n=676)\end{array}$ & $\begin{array}{l}0,05 \\
(n=675)\end{array}$ & $\begin{array}{l}-0,01 \\
(n=670)\end{array}$ & $\begin{array}{l}0,08 \\
(n=672)\end{array}$ & $\begin{array}{l}-0,02 \\
(n=675)\end{array}$ & $\begin{array}{l}0,03 \\
(n=660)\end{array}$ \\
\hline & Control de rabia & $\begin{array}{l}0,15^{* *} \\
(n=669)\end{array}$ & $\begin{array}{l}0,06 \\
(n=676)\end{array}$ & $\begin{array}{l}0,01 \\
(n=675)\end{array}$ & $\begin{array}{l}0,08 \\
(n=670)\end{array}$ & $\begin{array}{l}-0,02 \\
(n=672)\end{array}$ & $\begin{array}{l}0,19 * * * \\
(n=675)\end{array}$ & $\begin{array}{c}0,14^{*} \\
(n=660)\end{array}$ \\
\hline & $\begin{array}{l}\text { Expresión de rabia } \\
\text { compuesta }\end{array}$ & $\begin{array}{l}-0,21 * * * \\
(n=669)\end{array}$ & $\begin{array}{l}0,00 \\
(n=676)\end{array}$ & $\begin{array}{l}-0,03 \\
(n=675)\end{array}$ & $\begin{array}{l}-0,11 \\
(n=670)\end{array}$ & $\begin{array}{l}0,10 \\
(n=672)\end{array}$ & $\begin{array}{l}-0,13^{*} \\
(n=675)\end{array}$ & $\begin{array}{l}-0,15^{* *} \\
(n=660)\end{array}$ \\
\hline \multirow[t]{3}{*}{ DEQ } & Dependencia & $\begin{array}{l}-0,10 \\
(n=669)\end{array}$ & $\begin{array}{l}-0,01 \\
(n=676)\end{array}$ & $\begin{array}{l}0,15^{* *} \\
(n=675)\end{array}$ & $\begin{array}{l}-0,01 \\
(n=670)\end{array}$ & $\begin{array}{l}0,10 \\
(n=672)\end{array}$ & $\begin{array}{l}0,00 \\
(n=675)\end{array}$ & $\begin{array}{l}-0,02 \\
(n=660)\end{array}$ \\
\hline & Autocrítica & $\begin{array}{l}-0,36^{* * *} \\
(n=669)\end{array}$ & $\begin{array}{l}0,13^{*} \\
(n=676)\end{array}$ & $\begin{array}{l}-0,11 \\
(n=675)\end{array}$ & $\begin{array}{l}-0,14^{*} \\
(n=670)\end{array}$ & $\begin{array}{c}0,23^{* * *} \\
(n=672)\end{array}$ & $\begin{array}{l}-0,01 \\
(n=675)\end{array}$ & $\begin{array}{l}-0,21^{* * *} \\
(n=660)\end{array}$ \\
\hline & Autoeficacia & $\begin{array}{l}0,33^{* * *} \\
(n=669)\end{array}$ & $\begin{array}{l}0,21^{* * *} \\
(n=676)\end{array}$ & $\begin{array}{l}0,25^{* * * *} \\
(n=675)\end{array}$ & $\begin{array}{c}0,20 * * * \\
(n=670)\end{array}$ & $\begin{array}{l}0,07 \\
(n=672)\end{array}$ & $\begin{array}{l}0,09 \\
(n=675)\end{array}$ & $\begin{array}{r}0,31^{* * *} \\
(n=660)\end{array}$ \\
\hline
\end{tabular}

$* * * p<0,001 ; * * p<0,01 ; * p<0,05$.

Nota . SUPAF = Supervivencia y afrontamiento, MDESS $=$ Miedo a la desaprobación social, RESFAM = Responsabilidad con la familia, PREOC = Preocupación por los hijos, MSUIC = Miedo al suicidio, OBMOR = Objeciones morales, RFL = Escala total. Con $n=633$ a 676 , considerando los casos que respondieron completamente a las pruebas. 
El puntaje total de la escala RFL muestra correlaciones $(p<0,05)$ con la mayoría de las subescalas de los otros instrumentos utilizados, exceptuando el rasgo de rabia y la externalización de la rabia en STAXI y el estilo dependiente de la vivencia depresiva del DEQ. Las correlaciones encontradas son coherentes con lo esperable desde el punto de vista teórico y de la literatura, pues se ha observado que las personas presentan menor riesgo suicida en la medida que otorgan una mayor importancia a una mayor cantidad de razones para vivir (Echávarri, Morales et al., 2015), tienen un mayor bienestar subjetivo (Morales et al., 2017), una mayor satisfacción con el funcionamiento familiar (Lamis \& Lester, 2012), una experiencia de la rabia más funcional (Morales, Fischman et al., 2016) y un menor estilo autocrítico de vivencia depresiva (Morales et al., 2017).

Este mismo patrón de asociaciones es visible en particular con la subescala Supervivencia y afrontamiento. Es la escala que presenta una correlación más alta con el puntaje RFL total, compartiendo un mayor porcentaje de la varianza. Esta mayor atribución de importancia a las creencias en las propias capacidades de afrontamiento estaría asociada con una experiencia de mayor bienestar subjetivo (VerdugoLucero et al., 2012), con una mayor satisfacción con el funcionamiento familiar (Pavez, Santander, Carranza \& Vera-Villarroel, 2009), con un mejor manejo de la rabia (Morales, Fischman, et al., 2016) y con un estilo de vivencia depresiva de mayor eficacia y menor autocrítica (Morales, Echávarri et al., 2016).

Los resultados obtenidos de los análisis de la curva de ROC para distintos puntos de corte se pueden observar en la Tabla 8 y en la Figura 1.

Tabla 8

Puntos de Corte Propuestos para la Escala RFL Total con sus Respectivos Valores de Sensibilidad y Especificidad

\begin{tabular}{ccccc}
\hline Punto de corte & $\begin{array}{c}\text { Sensibilidad } \\
(\%)\end{array}$ & $\begin{array}{c}\text { Especificidad } \\
\text { (\%) }\end{array}$ & PPV (\%) & NPV (\%) \\
\hline 3,8 & 46,78 & 75,93 & 62,67 & 62,28 \\
3,9 & 52,14 & 71,29 & 61,08 & 63,28 \\
4,0 & 59,29 & 68,21 & 61,71 & 65,97 \\
4,1 & 62,50 & 65,12 & 60,76 & 66,77 \\
4,2 & 65,00 & 59,25 & 57,96 & 66,21 \\
\hline
\end{tabular}

Nota. Sensibilidad: Probabilidad de clasificar correctamente a una persona sin riesgo suicida; Especificidad: Probabilidad de clasificar correctamente a una persona con riesgo suicida; PPV: Probabilidad de detectar ausencia de riesgo suicida cuando efectivamente lo hay (falso negativo); NPV: Probabilidad de detectar presencia de riesgo suicida, cuando no lo hay (falso positivo). $N=643$, que corresponde a las personas que respondieron a todas las preguntas del set.

El área bajo la curva global ROC es de 65,8\%, 95\% IC [63,0\%, 67,3\%]. El punto de corte para el instrumento en su versión para Chile, que optimiza tanto la sensibilidad como la especificidad de la detección de casos en riesgo, es de 4,1. No obstante lo anterior, debe reconocerse que la prueba no es definitoria en la detección temprana, pues detecta dos tercios de los casos con riesgo de suicidio. Esta prueba se debe complementar con la entrevista clínica, una vez que arroje la presencia de riesgo. Es decir, se trata de una herramienta complementaria y no una única fuente de detección de situaciones de riesgo suicida.

Un puntaje total inferior a este valor sugeriría una valoración de motivos para vivir que ubican a las personas en situación de riesgo suicida. De esta forma, la prueba muestra un comportamiento adecuado para su uso como herramienta de tamizaje de riesgo suicida, asociando al punto de corte una sensibilidad de $62,10 \%$ y una especificidad es de $66,0 \%$ (Robin et al., 2011). Al usar el punto de corte sugerido por la literatura de 3,8 (García Valencia et al., 2009), se puede aumentar la especificidad a un 77,1\%, pero se baja la sensibilidad a un $47,5 \%$. El uso de uno u otro punto de corte dependerá de la aplicación que se quiera dar a la prueba. 


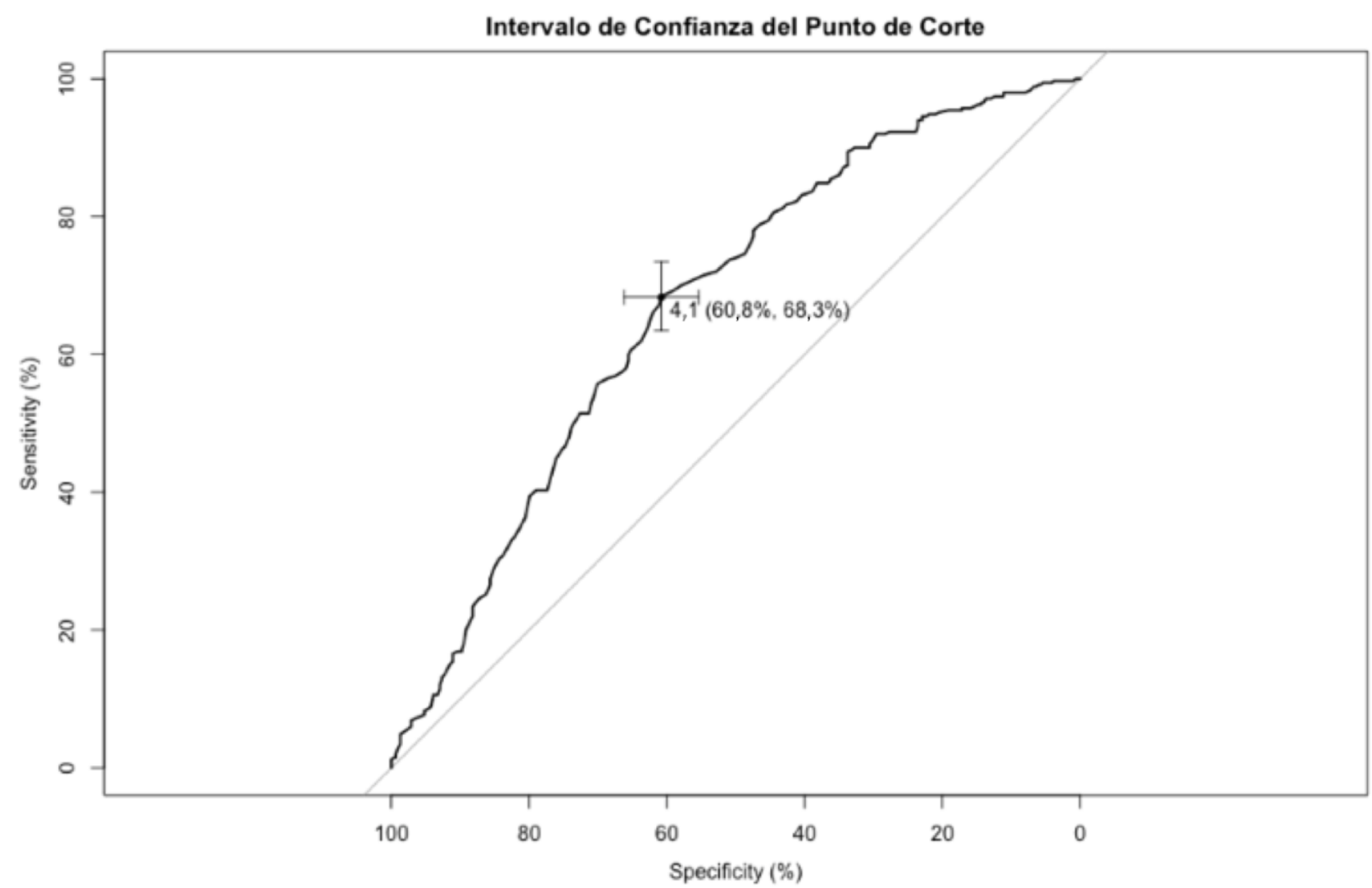

Figura 1. Curva ROC de punto de corte de la escala RFL. El área bajo la curva global es de 65,8\%, lo que significa que la prueba logra clasificar correctamente la ausencia de riesgo a ese porcentaje de los consultantes, con un intervalo de confianza de 95\% [63,0\%, 67,3\%] de clasificación correcta en la población consultante a servicios de salud mental.

\section{Discusión}

En el presente trabajo se presentó la validación para Chile de la escala RFL que evalúa la importancia atribuida a las razones para mantenerse con vida cuando se piensa en el suicidio (Linehan et al., 1983). Esta escala, en su traducción al español, presenta una estructura factorial similar a la escala original y las subescalas son estables internamente (García Valencia et al., 2009). Esto es congruente con lo presentado en el estudio original de Linehan et al. (1983) y con otros estudios de la RFL realizados en Latinoamérica (Cassaretto \& Martínez, 2012).

En términos generales, los resultados muestran propiedades psicométricas adecuadas en cuanto a consistencia interna, estructura factorial general y capacidad discriminativa. Sin embargo, a diferencia de los seis factores correspondientes a la estructura teórica propuesta para el instrumento original (Cassaretto \& Martínez, 2012; García Valencia et al., 2009; Linehan et al., 1983), los resultados permitieron detectar cinco factores. La diferencia en la muestra chilena es que no logra distinguirse la subescala Miedo al suicidio de la subescala Miedo a la desaprobación social. Estos resultados orientan a que en esta población puede ser más adecuado una solución unidimensional que una separación por subescalas.

Con respecto a la validez concurrente, el puntaje total de la escala RFL muestra correlaciones con la mayoría de las subescalas y escalas clínicas aplicadas a la muestra. Es así como a mayor puntaje en la RFL mayor es el sentimiento de bienestar en cuanto a sintomatología ansiosa y depresiva, calidad de las relaciones interpersonales y adecuación en el rol social. También es mayor la satisfacción con el funcionamiento familiar, es menor el estilo autocrítico de vivencia depresiva y la experiencia de rabia es más funcional. Se destaca la subescala Supervivencia y afrontamiento, ya que presenta una mayor correlación con el puntaje RFL total, compartiendo un mayor porcentaje de la varianza. Esto refuerza la idea de que la confianza en las propias 
capacidades para manejar los problemas y las situaciones estresantes protege del riesgo suicida, siendo un aspecto a fortalecer en las intervenciones terapéuticas (Barros et al., 2017).

Para la población clínica chilena se propone un punto de corte de la RFL de 4,1 puntos en la escala total. Esto puede servir de advertencia a los tratantes para indagar clínicamente y en mayor profundidad el riesgo suicida pesquisado en el momento. Especialmente, si consideramos que el estado de riesgo suicida es altamente fluctuante (Fowler, 2012), la detección del riesgo actual es una oportunidad para profundizar e intervenir en ese momento crítico. Cabe mencionar que el porcentaje de falsos negativos es alto, por lo que se resalta aun más la importancia de complementar con la evaluación clínica y aprovechar los aportes cualitativos de la escala para la intervención.

En cuanto a la aplicación terapéutica de los resultados de la escala RFL, además de señalar la probabilidad de presentar riesgo suicida actual, permite identificar recursos y aspectos que el consultante valora para mantenerse con vida. Al mismo tiempo, es posible visualizar aquellas áreas más vulnerables, que pueden ser fortalecidas a través de psicoterapia. Accediendo al detalle de los ítems, se pueden identificar los aspectos personales, interpersonales y valóricos que disuaden de cometer un intento suicida. En el presente estudio esto se destaca en el caso de los ítems asociados a la subescala Supervivencia y afrontamiento. Al respecto, reviste especial importancia identificar los recursos en los consultantes y considerarlos para la recuperación del sentido de la vida. Lo anterior puede aumentar el éxito y eficacia de un proceso psicoterapéutico (Echávarri, Morales et al., 2015), mediante el reforzamiento de las razones para vivir. Este enfoque en la intervención podría conducir a experimentar un mayor sentimiento de bienestar en cuanto a la sintomatología ansiosa y depresiva, calidad de las relaciones interpersonales y adecuación en el rol social, una mayor satisfacción con el funcionamiento familiar, una experiencia de la rabia más funcional y un menor estilo autocrítico de vivencia depresiva.

Los resultados de esta investigación son similares a los encontrados en otros países (Cassaretto \& Martínez, 2012; García-Valencia et al., 2007) en relación a los motivos considerados más importantes para no suicidarse. Es importante desarrollar nuevos estudios longitudinales que permitan determinar la magnitud del efecto en los resultados terapéuticos e intervenir en el fortalecimiento de las razones para vivir y otros recursos personales.

El presente estudio consideró una muestra de un amplio rango de edad, desde adolescentes hasta adultos mayores, y las razones para vivir pueden tener valoraciones diferentes según la etapa de vida en que se encuentra la persona. Al respecto, los resultados sugieren que no habría diferencias en relación a las dimensiones generales. Las razones disuasivas del suicidio más importantes son las relacionadas con la familia y con las creencias acerca de las propias capacidades para enfrentar los problemas. Sin embargo, de los 705 participantes, 603 (85,53\%) pertenecen al rango adulto, entre 18 y 65 años, por lo que los resultados son aplicables especialmente a consultantes en este rango de edad. Podría ser de utilidad validar las versiones de la escala RFL para otros grupos de edad, particularmente adolescentes, dada la reconocida importancia para la salud pública de Chile la prevención del suicidio en este subgrupo (MINSAL, 2011).

Este estudio se enfocó en consultantes a servicios de salud mental de la Región Metropolitana de Chile que mayoritariamente tenían diagnóstico de trastorno del ánimo. Estos últimos son los que presentan una mayor asociación con el suicidio consumado (MINSAL, 2013). En estudios de autopsia psicológica se muestra que un $90 \%$ de las personas que fallecieron por suicidio presentaba un cuadro psiquiátrico diagnosticado y un $80 \%$ de ellas era de tipo depresivo (WHO, 2014). No obstante, los resultados de este estudio no son generalizables a la población general o de otras regiones del país. Tampoco lo son respecto de consultantes con otros diagnósticos.

La población que asiste a servicios de salud mental muestra una gran variedad de motivos de consulta y perfiles clínicos, por lo que el desafío del especialista es evaluar, ponderar y discriminar quién puede presentar mayor riesgo de suicidio y quien no, ya que esto implica decisiones de tratamiento e indicaciones de cuidado diferentes. El aporte de la validación de la RFL y el punto de corte propuesto para Chile es solo un complemento al juicio clínico y a la distinción en una población que ya se supone que es de mayor riesgo, por tratarse de una población consultante a salud mental (WHO, 2014).

Dado que el proceso de evaluación estuvo sujeto a la aceptación voluntaria de participar, hubo algunos consultantes que, cumpliendo con los criterios para ser incluidos en el estudio, no estarían representados en los resultados obtenidos. Asimismo, la muestra finalmente quedó conformada por una mayoría de mujeres, reflejando la tendencia que se observa generalmente en los consultantes a salud mental. Sería importante 
incluir en los futuros estudios a participantes de la población general, analizando las diferencias con la población clínica.

Si bien en este estudio se correlacionaron los puntajes de la RFL con otras escalas relacionadas con el riesgo suicida, sería de utilidad hacer análisis comparativos con otras escalas de riesgo suicida ya validadas en Chile, como el Inventario de Depresión de Beck y la Escala de Suicidalidad de Okasha (Okasha, Lotaif, \& Sadek, 1981; Salvo, Melipillán \& Castro, 2009; Valdés et al., 2017). De esta forma, se podrían obtener resultados más válidos en relación a la población chilena.

Considerando las limitaciones señaladas, se destaca que ahora se cuenta con la escala RFL estudiada y con valores psicométricos en población clínica chilena, constituyendo un aporte tanto clínico como investigativo. Esta escala se distingue por su capacidad de pesquisar un probable riesgo suicida actual a partir de la evaluación de las propias razones para vivir. Se complementa con las evaluaciones específicas de los pensamientos y conductas suicidas, aportando los recursos a fortalecer en la intervención terapéutica. Es importante destacar que el resultado de la evaluación, al momento de aplicar el instrumento, es una advertencia a los tratantes para indagar clínicamente y en mayor profundidad el riesgo suicida detectado.

\section{Referencias}

Appleby, L., Shaw, J., Amos, T., McDonnell, R., Harris, C., McCann, K. Parsons, R. (1999). Suicide within 12 months of contact with mental health services : National clinical survey. BMJ British Medical Journal, 318, 1235-1239. https://doi.org/10.1136/bmj.318.7193.1235

Baader-Matthei, T., Richter, P. \& Mundt, C. (2004). Suicidios de pacientes psiquiátricos hospitalizados y sus factores de riesgo. Un estudio caso control. Revista Chilena de Neuro-Psiquiatría, 42, 293-316. https://doi.org/10.4067/S0717-92272004000400006

Barros, J., Morales, S., Echávarri, O., García, A., Ortega, J., Asahi, T. Núñez, C. (2017). Suicide detection in Chile: Proposing a predictive model for suicide risk in a clinical sample of patients with mood disorders. Revista Brasileira de Psiquiatria, 39, 1-11. https://doi.org/10.1590/1516-4446-2015-1877

Blatt, S. J., D'Afflitti, J. P. \& Quinlan, D. M. (1976). Experiences of depression in normal young adults. Journal of Abnormal Psychology, 85, 383-389. https://doi.org/10.1037/0021-843X.85.4.383

Bobes García, J., Giner Ubago, J. \& Saiz Ruiz, J. (Eds.) (2011). Suicidio y psiquiatría: recomendaciones preventivas y de manejo del comportamiento suicida. Madrid, España: Fundación Española de Psiquiatría y Salud Mental.

Borowsky, I. W., Resnick, M. D., Ireland, M. \& Blum, R. W. (1999). Suicide attempts among American Indian and Alaska native youth: Risk and protective factors. Archives of Pediatrics \& Adolescent Medicine, 153, 573-580. https://doi.org/10.1001/archpedi.153.6.573

Cassaretto, M. \& Martínez, P. (2012). Razones para vivir en jóvenes adultos: validación del RFL-YA. Revista de Psicología, Pontificia Universidad Católica del Perú, 30, 170-188. Extraído de http://pepsic.bvsalud.org/scielo.php?script=sci_arttext\&pid=S025492472012000100007

Chile, Ministerio de Salud (2011). Metas 2011-2020: elige vivir sano. Estrategia Nacional de Salud para el cumplimiento de los objetivos sanitarios de la década 2011-2020. Santiago, Chile: Autor. Extraído de http://www.ispch.cl/sites/default/files/documento/2011/12/Metas20112020.pdf

Chile, Ministerio de Salud (2013). Programa Nacional de Prevención del Suicidio: orientaciones para su implementación. Santiago, Chile: Autor. Extraído de http://web.minsal.cl/sites/default/files/Programa_Nacional_Prevencion.pdf

Dobrov, E. \& Thorell, L. H. (2004). Reasons for living: Translation, psychometric evaluation and relationships to suicidal behaviour in a Swedish random sample. Nordic Journal of Psychiatry, 58, 277-285. https://doi.org/10.1080/08039480410005783

Echávarri, O., Fischman, R., Morales, S., Murillo, C. \& Barros, J. (2015). ¿Qué debemos evaluar en consultantes de salud mental con riesgo suicida? Gaceta de Psiquiatría Universitaria, 11, 72-79. Extraído de http://revistagpu.cl/2015/GPU_marzo_2015_PDF/PC_Que_debemos.pdf

Echávarri, O., Maino, M. P., Fischman, R., Morales, S. \& Barros, J. (2015). Aumento sostenido del suicidio en Chile: un tema pendiente. Temas de La Agenda Pública, 10, artículo 79. Extraído de http://politicaspublicas.uc.cl/wp-content/uploads/2015/07/N\%C2\%B0-79Aumento-sostenido-del-suicidio-en-Chile.pdf

Echávarri, O., Morales, S., Bedregal, P., Barros, J., Maino, M. P., Fischman, R. Moya, C. (2015). ¿Por qué no me suicidaría? Comparación entre pacientes hospitalizados en un servicio de psiquiatría con distinta conducta suicida. Psykhe, 24(1), 1-11. https://doi.org/10.7764/psykhe.24.1.667

Edelstein, B. A., Heisel, M. J., McKee, D. R., Martin, R. R., Koven, L. P., Duberstein, P. R. \& Britton, P. C. (2009). Development and psychometric evaluation of the Reasons for Living-Older Adults Scale: A suicide risk assessment inventory. The Gerontologist, 49, 736-745. https://doi.org/10.1093/geront/gnp052

Forgays, D. G., Forgays, D. K. \& Spielberger, C. D. (1997). Factor structure of the State-Trait Anger Expression Inventory. Journal of Personality Assessment, 69, 497-507. https://doi.org/10.1207/s15327752jpa6903_5

Fowler, J. C. (2012). Suicide risk assessment in clinical practice: Pragmatic guidelines for imperfect assessments. Psychotherapy, 49, 8190. https://doi.org/10.1037/a0026148

García-Valencia, J., Palacio-Acosta, C., Arias, S., Ocampo, M., Calle, J., Restrepo, D. López, C. (2007). Características asociadas al riesgo de suicidio valorado clínicamente en personas con intento reciente. Revista Colombiana de Psiquiatría, 36, 610-627. Extraído de http://www.scielo.org.co/pdf/rcp/v36n4/v36n4a03

García Valencia, J., Palacio Acosta, C. A., Vargas, G., Arias, S., Ocampo, M. V., Aguirre, B. García, H. (2009). Validación del "inventario de razones para vivir" (RFL) en sujetos con conducta suicida de Colombia. Revista Colombiana de Psiquiatría, 38, 66-84. Extraído de http://www.scielo.org.co/scielo.php?script=sci_arttext\&pid=S0034-74502009000100006

Gargurevich, R., Luyten, P. \& Corveleyn, J. (2008). Dependency, self criticism, social support and posttraumatic stress disorder symptoms in Peruvian university students. International Journal of Psychology, 43, $435 . \quad$ Extraído de http://discovery.ucl.ac.uk/1311569/

Kline, R. B. (2011). Principles and practice of structural equation modeling. (3a ed.). New York, NY: Guilford Press. 
Lambert, M. J., Burlingame, G. M., Umphress, V., Hansen, N. B., Vermeersch, D. A., Clouse, G. C. \& Yanchar, S. C. (1996). The reliability and validity of the Outcome Questionnaire. Clinical Psychology \& Psychotherapy, 3, 249-258. https://doi.org/10.1002/(SICI)10990879(199612)3:4<249::AID-CPP106>3.0.CO;2-S

Lamis, D. A. \& Lester, D. (2012). Risk and protective factors for reasons for living in college men. International Journal of Men's Health, 11, 189-201. https://doi.org/10.3149/jmh.1103.189

Leibovich de Figueroa, N. B., Schmidt, V. \& Gol, S. (2001). El Inventario de Expresión de Enojo Estado-Rasgo (STAXI) y su uso en diferentes poblaciones. Revista Iberoamericana de Diagnóstico y Evaluación Psicológica, 11, 55-74. Extraído de http://www.aidep.org/03_ridep/R11/R113.pdf

Linehan, M., Goodstein, J., Nielsen, S. L. \& Chiles, J. (1983). Reasons for staying alive when you are thinking of killing yourself: The Reasons for Living Inventory. Journal of Consulting and Clinical Psychology, 51, 276-286. https://doi.org/10.1037/0022006X.51.2.276

Maddaleno, M., Horwitz, N., Jara Vargas, C., Florenzano Urzúa, R. \& Zalazar, D. (1987). Aplicación de un instrumento para calificar el funcionamiento familiar en la atención de adolescentes. Revista Chilena de Pediatría, 58, 246-249. Extraído de http://bases.bireme.br/cgibin/wxislind.exe/iah/online/?IsisScript=iah/iah.xis\&src=google\&base=ADOLEC\&lang=p\&nextAction=lnk\&exprSearch=58702\&in dexSearch=ID

Manzini, J. L. (2000). Declaración de Helsinki: principios éticos para la investigación médica sobre sujetos humanos. Acta Bioethica, 6, 321-334. https://doi.org/10.4067/S1726-569X2000000200010

Morales, S., Echávarri, O., Barros, J., Fischman, R., Zuloaga, F., Taylor, T. \& Peñaloza, F. (2015). Pacientes hospitalizados por riesgo suicida: ¿cómo perciben la ayuda recibida? Revista Argentina de Clínica Psicológica, 24, 67-78. Extraído de http://www.academia.edu/15225457/Pacientes_Hospitalizados_por_Riesgo_Suicida_C\%C3\%B3mo_perciben_la_ayuda_recibida

Morales, S., Echávarri, O., Barros, J., Maino, M. P, Armijo, I., Fischman, R. Monari, M. (2017). Intento e ideación suicida en consultantes a salud mental: estilos depresivos, malestar interpersonal y satisfacción familiar. Psykhe, 26(1), 1-15. https://doi.org/10.7764/psykhe.26.1.939

Morales, S., Echávarri, O., Barros, J., Zuloaga, F. \& Taylor, T. (2016). Percepción del propio riesgo suicida: estudio cualitativo con pacientes hospitalizados por intento o ideación suicida. Revista Argentina de Clínica Psicológica, 25, 245-258. Extraído de http://www.clinicapsicologica.org.ar/numero.php?idn=31

Morales, S., Fischman, R., Echávarri, O., Barros, J., Armijo, I., Moya, C. Núñez, C. (2016). Vivencia-expresión de la rabia y razones para vivir en un grupo de pacientes chilenos con riesgo suicida. Revista Iberoamericana de Psicología y Salud, 7, 60-68. https://doi.org/10.1016/j.rips.2016.03.002

Okasha, A., Lotaif, F. \& Sadek, A. (1981). Prevalence of suicidal feelings in a sample of non-consulting medical students. Acta Psychiatrica Scandinavica, 63, 409-415. https://doi.org/10.1111/j.1600-0447.1981.tb00690.x

Organisation for Economic Co-operation and Development (2015). Health at a glance 2015: OECD indicators. Paris, Francia: Autor. https://doi.org/10.1787/health_glance-2015-1-en

Organización Mundial de la Salud (2012). Prevención del suicidio (SUPRE). Genève, Suiza: Autor. Extraído de http://www.who.int/mental_health/prevention/suicide/suicideprevent/es/

Organización Panamericana de la Salud (2003). Informe mundial sobre la violencia y la salud (Publicación Científica y Técnica $\mathrm{N}^{\circ} 588$ ). Washington, DC: Autor. Extraído de http://apps.who.int/iris/bitstream/handle/10665/112670/9275315884_spa.pdf?sequence=1Organización Panamericana de la Salud (2014). Mortalidad por suicidio en las Américas (Informe regional). Washington, DC: Autor. Extraído de http://www.bvsde.paho.org/documentosdigitales/bvsde/texcom/PAHOMortalidad-suicidio.pdf

Osman, A., Kopper, B. A., Linehan, M. M., Barrios, F. X., Gutierrez, P. M. \& Bagge, C. L. (1999). Validation for the Adult Suicidal Ideation Questionnaire and the Reasons for Living Inventory in an adult psychiatric inpatient sample. Psychological Assessment, 11, 115-123. https://doi.org/10.1037/1040-3590.11.2.115

Pavez, P., Santander, N., Carranza, J. \& Vera-Villarroel, P. (2009). Factores de riesgo familiares asociados a la conducta suicida en adolescentes con trastorno depresivo. Revista Médica de Chile, 137, 226-233. https://doi.org/10.4067/S0034-98872009000200006

$\mathrm{R}$ Core Team (2016). R: A language and environment for statistical computing. Wien, Austria: R Foundation for Statistical Computing.

Robin, X., Turck, N., Hainard, A., Tiberti, N., Lisacek, F., Sanchez, J.-C. \& Müller, M. (2011). pROC: An open-source package for R and S+ to analyze and compare ROC curves. BMC Bioinformatics, 12, Artículo 77. https://doi.org/10.1186/1471-2105-12-77

Salvo, L., Melipillán, R. \& Castro, A. (2009). Confiabilidad, validez y punto de corte para escala de screening de suicidalidad en adolescentes. Revista Chilena de Neuro-Psiquiatría, 47, 16-23. http://dx.doi.org/10.4067/S0717-92272009000100003

Smilkstein, G. (1978). The family APGAR: A proposal for a family function test and its use by physicians. The Journal of Family Practice, 6, 1231-1239. Extraído de http://psycnet.apa.org/psycinfo/1979-26481-001

Taylor, T., Morales, S., Zuloaga, F., Echávarri, O. \& Barros, J. (2012). Lo que nos dicen los padres: perspectivas de los padres de pacientes hospitalizados por ideación o intento suicida. Revista Argentina de Clínica Psicológica, 21, 271-280. Extraído de http://www.redalyc.org/articulo.oa?id=281929021008

Valdés, C., Morales-Reyes, I., Pérez, J. C., Medellín, A., Rojas, G. \& Krause, M. (2017). Propiedades psicométricas del Inventario de Depresión de Beck IA para la población chilena. Revista Médica de Chile, 145, 1005-1012. https://doi.org/10.4067/s003498872017000801005

Verdugo-Lucero, J. C., Ponce de León-Pagaza, B. G., Guardado-Llamas, R. E., Meda-Lara, R. M., Uribe-Alvarado, J. I. \& Guzmán-Muñiz, J. (2012). Estilos de afrontamiento al estrés y bienestar subjetivo en adolescentes y jóvenes. Revista Latinoamericana de Ciencias Sociales, Ninez y Juventud, 11, 79-91. https://doi.org/10.11600/1692715x.1114120312

von Bergen, A. \& de la Parra, G. (2002). OQ-45.2, cuestionario para evaluación de resultados y evolución en psicoterapia: adaptación, validación e indicaciones para su aplicación e interpretación. Terapia Psicológica, 20, 161-176. Extraído de https://www.researchgate.net/profile/Alejandra_Von_Bergen/publication/263314562_OQ45.2_CUESTIONARIO_PARA_EVALUACIN_DE_RESULTADOS_Y_EVOLUCIN_EN_PSICOTERAPIA_ADAPTACIN_VALIDACIN_E_INDICAC IONES_PARA_SU_APLICACIN_E_INTERPRETACIN_OQ-45.2_AN_OUTCOME_QUESTIONNA

Woods, A. M., Zimmerman, L., Carlin, E., Hill, A. \& Kaslow, N. J. (2013). Motherhood, reasons for living, and suicidality among African American women. Journal of Family Psychology, 27, 600-606. https://doi.org/10.1037/a0033592

World Health Organization (2014). Preventing suicide: A global imperative. Luxembourg, Luxemburgo: Autor. Extraído de http://apps.who.int/iris/bitstream/10665/131056/1/9789241564779_eng.pdf 


\section{Anexo}

\section{Razones para Vivir \\ Instrumento Traducido al Español}

Nombre:

Instrucciones:

Muchas personas han pensado en el suicidio alguna vez. Otras nunca lo han considerado. Independiente de si lo ha considerado o no, nos interesan las razones que usted tendría para no suicidarse si alguna vez se le ocurriera hacerlo, o si alguien se lo sugiriera.

En las siguientes páginas hay razones que las personas a veces dan para no suicidarse. Queremos saber cuán importantes son cada una de estas posibles razones para usted, en este momento de su vida, como una razón para no suicidarse. Por favor clasifíquelas en el espacio a la izquierda de cada pregunta.

Cada razón puede clasificarse desde 1 (Para nada importante) a 6 (Extremadamente importante). Si una razón no se aplica para usted, o si no piensa que la afirmación sea verdadera, entonces probablemente no es importante y debiera poner un 1. Por favor utilice el rango completo de opciones para no calificar solamente en el medio $(2,3,4,5)$ o solamente en los extremos $(1,6)$.

En cada espacio ponga un número para indicar la importancia que tiene para Ud. cada razón para no suicidarse.

1. Para nada importante (como una razón para no suicidarse, o, no se aplica para mí, no creo para nada en esto).

2. Muy poco importante

3. Poco importante

4. Algo importante

5. Bastante importante

6. Extremadamente importante (como una razón para no suicidarme, creo mucho en esto y es muy importante).

Incluso si nunca lo ha considerado, o cree firmemente que nunca consideraría el suicidio de forma seria, aun así, es importante que califique cada razón. En este caso, califique basándose en por qué suicidarse no es o nunca sería una alternativa para Ud.

En cada espacio ponga un número para indicar la importancia que tiene para Ud. cada razón para no suicidarse.

1. Para nada importante

2. Muy poco importante

3. Poco importante
4. Algo importante

5. Bastante importante

6. Extremadamente importante

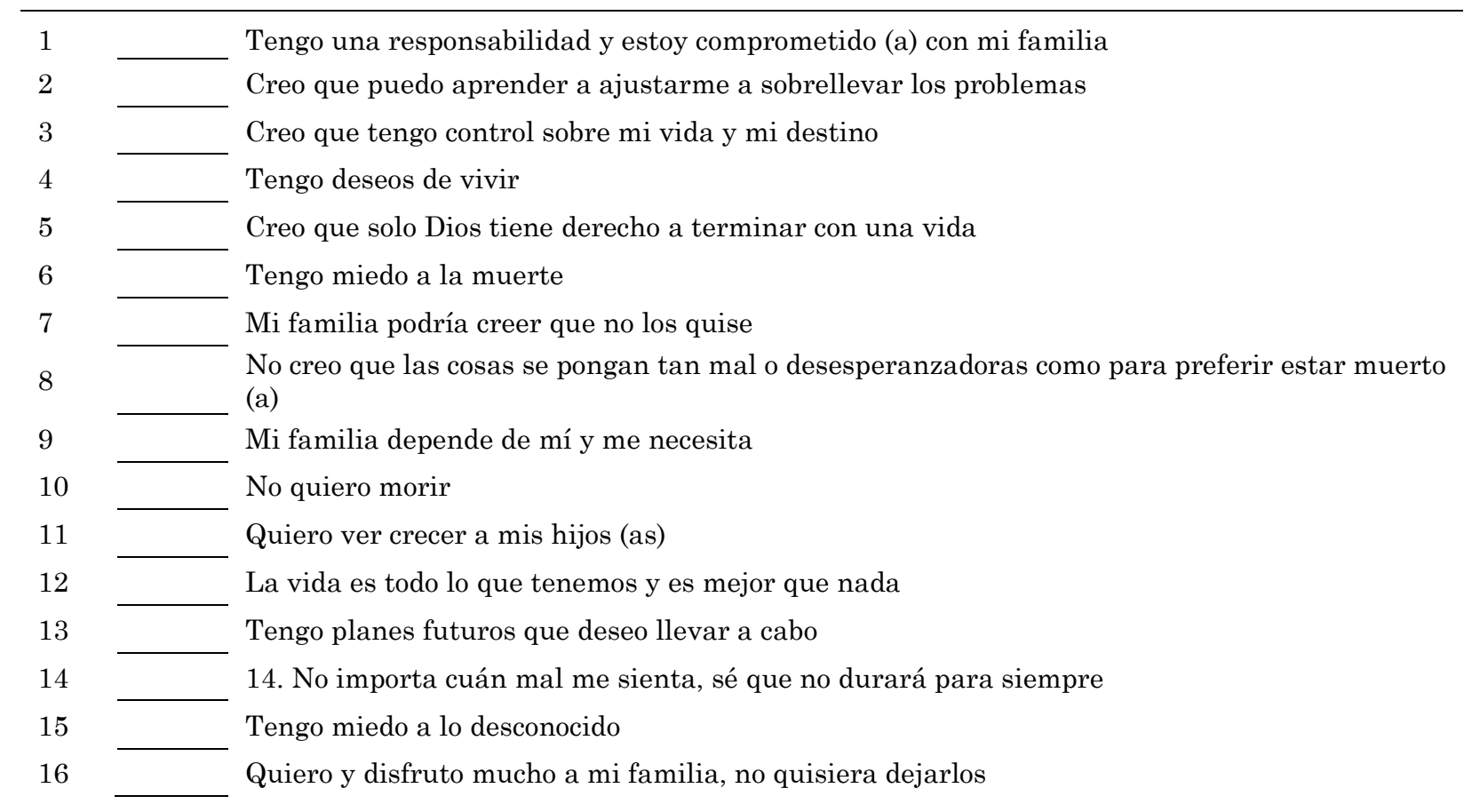




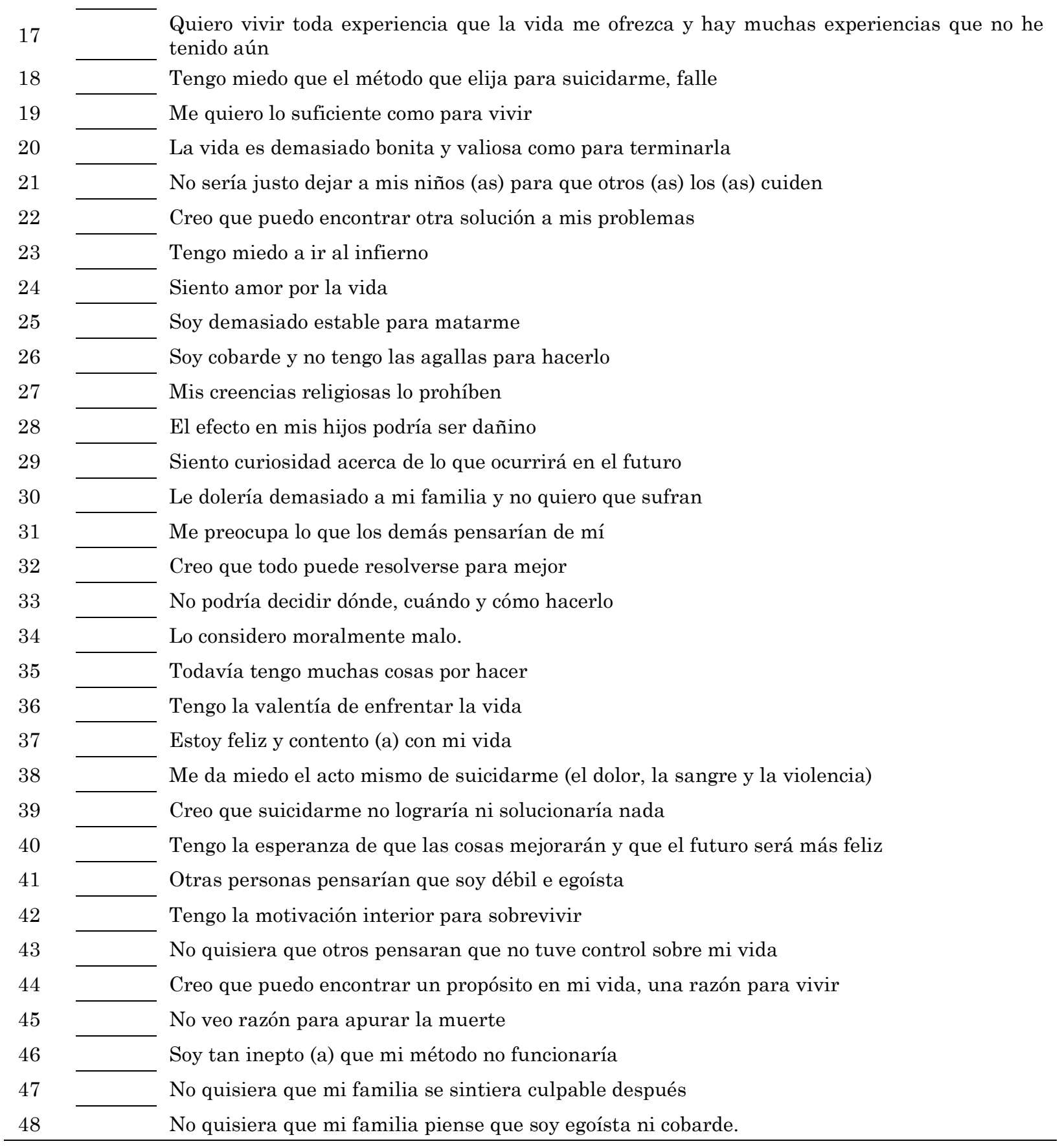

Fecha de recepción: Enero de 2017.

Fecha de aceptación: Enero de 2018. 